\title{
Training in robotic thoracic surgery-the European way
}

\author{
Ghada M. M. Shahin ${ }^{1}$, George J. Brandon Bravo Bruinsma ${ }^{1}$, Sasha Stamenkovic ${ }^{2}$, Miguel A. Cuesta ${ }^{3}$ \\ ${ }^{1}$ Department of Cardiothoracic Surgery, Isala Heart Centre, Zwolle, The Netherlands; ${ }^{2}$ The Thorax Centre, St. Bartholomew's Hospital, London, \\ UK; ${ }^{3}$ Department of General Surgery, Vrije Universiteit Medisch Centrum, Amsterdam, The Netherlands \\ Correspondence to: Ghada M. M. Shahin, MD. Isala Heart Centre, Dr. Van Heesweg 2, 8025 AB Zwolle, The Netherlands. \\ Email: g.m.m.shahin@isala.nl.
}

\begin{abstract}
The increasing demand for robot-assisted thoracic surgery (RATS) in Europe requires a structured and standardized training module. Until now, Intuitive Surgical Inc. (Sunnyvale, CA, USA) has delivered the only available robotic surgery platform. Although the training program that is organized by Intuitive is divided in an initial and an advanced course, the success of the training depends on many external factors. Until now the training focused on experienced thoracic surgeons. The aim of this article is to offer a stepwise training module, which can be adopted by experienced open (thoracotomy) surgeons or video-assisted thoracic (VATS) surgeons but is primarily meant for thoracic surgery fellows and residents, as it is our sincere opinion that we should focus on training for this type of surgery as early in their careers as possible. In order to maintain surgical technique and minimize the chance of complications, on-going training and certification of the surgeons and the team is deemed necessary.
\end{abstract}

Keywords: Robotic thoracic surgery; training; simulation; video-assisted thoracoscopic surgery (VATS)

Submitted Aug 21, 2018. Accepted for publication Oct 04, 2018.

doi: $10.21037 /$ acs.2018.11.06

View this article at: http://dx.doi.org/10.21037/acs.2018.11.06

\section{Introduction}

Video-assisted thoracoscopic surgery (VATS) for major pulmonary resections has being increasingly implemented since its introduction in the late nineties (1). The evolution of VATS lobectomy from multi- to uni-portal VATS demonstrates technical progress, for example, improved camera optics allowing clear visualization and articulating staplers and roticulator instruments that allow the surgical fulcrum to be more inside the chest. This creates conditions that are similar to open thoracotomy (2). However, the teaching of this technique remains demanding (3).

Robot-assisted surgery is an evolving technique, comprising 4,271 operational Intuitive Surgical da Vinci ${ }^{\mathrm{TM}}$ systems worldwide up to September 30, 2017. Over the past five years, thoracic surgery has been focused on using robotics as a novel minimally invasive technique to perform major lung resections and resections of mediastinal masses (4). Consequently, there is an increasing demand for a structured, standardized training module (5).

Several authors have demonstrated the feasibility and safety of RATS for thymectomy, lobectomy, sleeve lobectomy, segmentectomy and pneumonectomy for lung cancer and a variety of other complex thoracic procedures (6-8). All agree that surgery of the closed chest requires thorough knowledge of the anatomy and specific dexterities to cope with life-threatening complications. Therefore, at this moment, only experienced thoracic surgeons are being trained. In contrast to VATS, robotic surgery has many features that allow expeditious and safe teaching of this technique. Having said that, we can focus on training young surgeons such as fellows and residents in order to integrate the robotic training in the overall surgical residency, as is already happening in the United States (9).

\section{Developments}

Ever since the release of the da Vinci $S^{\mathrm{TM}}$ system in 2000, the training to become a console (operating) surgeon has shifted from mostly self-taught surgeons to surgeons who are subjected to a defined training facilitated by Intuitive Surgical Inc. so they can perform reliable and safe 


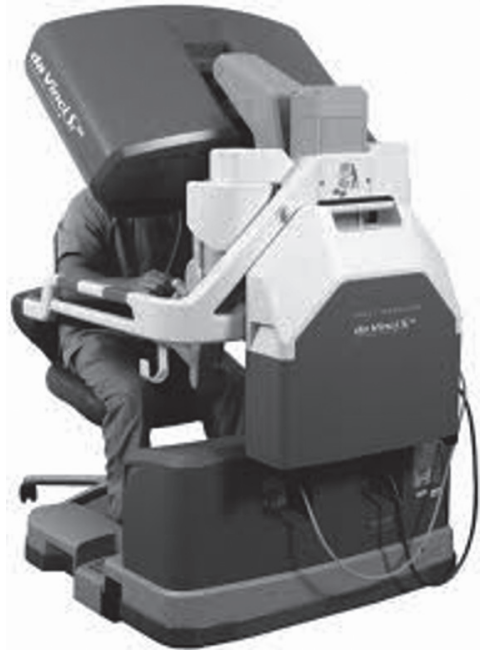

Figure 1 da Vinci Skill Simulator.

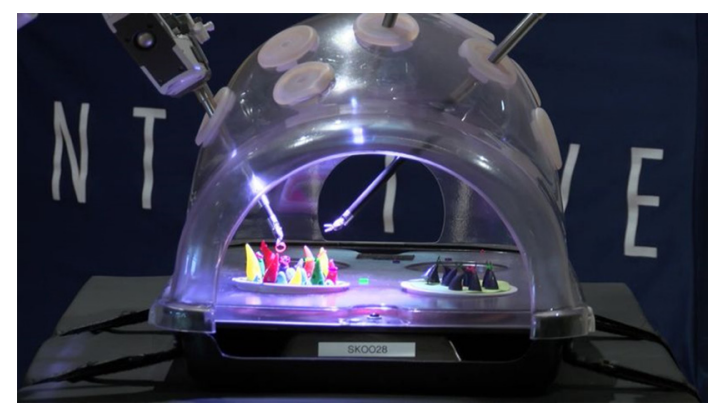

Figure 2 Plastic model for exercising port placement, docking, undocking and simple dexterity.

procedures. Further developments have led to the Si system and most recently the $\mathrm{Xi}$ and $\mathrm{X}$ system with improved exercises available on the da Vinci Skill Simulator (Figure 1). The plastic model (Figure 2) serves its purpose especially for port placement, docking and undocking. E-learning modules are available for all systems. A recent development by Kindheart (www.Kindheart.com) is a simulation model with real animal/porcine tissue. These models can be used for a variety of operations and allow more realistic surgery than the da Vinci Skills Simulator. We quote the Kindheart website: "The Thoracic Surgery Simulator (TSS) is used with the Kindheart Thoracic Patient and Kindheart Thoracic Tissue Cassette to enable simulation of thoracic surgery on a human patient. The cassette is inserted into a Kindheart Thoracic Patient which simulates a buman patient from shoulder to bip in a left lateral position. Surgery can be performed using open, laparoscopic, or robotic techniques." Unquote.
These developments urge us to rethink the current training model with expensive porcine and cadaver models.

The following describes the early introduction of the robotic surgery program in the lead author's cardiothoracic unit of a university-affiliated, tertiary top-clinical hospital in the Netherlands and is followed by a stepwise approach for successful implementation.

\section{The training program (RATS) in Isala Heart Centre in 2011}

In Table 1, we describe the chronological sequence of how our training was set up with the da Vinci Si system from the development and approval of the business case until the training of own staff members today.

As the "go ahead" signal of the Board of Directors was received, the clinical sales representative (CSR) of Intuitive started training the team with multiple dry-runs using the plastic model. The team was stimulated to acquire thorough knowledge of the "buttonology", the available instruments and their function. System troubleshooting was practiced and an emergency protocol designed. These procedures and protocols were learned by heart and practiced many times. The team was instructed during human cadaver training in the Ecole Européenne de Chirurgie (EEC) in Paris, France. A technician of Intuitive guided us in port placement, docking of the patient cart and emergency undocking. Finally, a certificate of competence was issued. Back home, "dry practicing" continued whenever time permitted. Simple thoracic procedures such as bullectomy, pleurectomy, and pulmonary wedge resections were performed without proctor.

At that time, in 2011, we had to cope with little experience in robotic thoracic surgery in Europe and thus few proctors. This turned out in our favor as we got the chance to work with the pioneers and experts (F Melfi and $\mathrm{R}$ Cerfolio) in this field.

Two major drawbacks were that the simulator was purchased one year later. As we realize the importance of simulator training now, the simulator should have been purchased earlier. Furthermore, a dual console system is until today not available for our unit.

Another lesson we have learned is that a robotic thoracic program run by only one console surgeon and one bedside surgeon can be a vulnerable situation. It was therefore decided, shortly after the initial learning curve, to train other staff members. It is from that experience that we recommend starting with the training of two console 


\begin{tabular}{|c|c|c|}
\hline Business case & Depts. of Cardiothoracic Surgery and Urology & 2010-2011 \\
\hline Case observation (Prof. Dr. F Melfi, Pisa, Italy) & CSR, surgeon, anaesthetist, scrub nurse & Sep. 2011 \\
\hline Cadaver training & CSR, surgeons, scrub nurses & Sep. 2011 \\
\hline Development of emergency protocol & Team & Oct. 2011 \\
\hline $\begin{array}{l}\text { Lobectomy case } 1-5 \text {, } \\
\text { proctored by Prof. Dr. F Melfi }\end{array}$ & $\begin{array}{l}\text { Team (one console surgeon, one bedside surgeon, } \\
\text { two scrub nurses, one anaesthetist and one } \\
\text { anaesthesia technician) }\end{array}$ & Nov. 2011 to Feb. 2012 \\
\hline $\begin{array}{l}\text { "Advanced" course (Prof. Dr. R Cerfolio, UAB, } \\
\text { Birmingham, USA) }\end{array}$ & $\begin{array}{l}\text { Team (two surgeons, two scrub nurses and one } \\
\text { nurse of the Pulmonology Department) }\end{array}$ & Jan. 2012 \\
\hline Purchase of simulator & Depts. of Cardiothoracic Surgery and Urology & 2012 \\
\hline Teaching other staff members & Lead author of this manuscript & 2014 to today \\
\hline $\begin{array}{l}\text { Proctoring for surgeon led courses and } \\
\text { surgery on site }\end{array}$ & Lead author of this manuscript & 2015 to today \\
\hline
\end{tabular}

RATS, robot-assisted thoracic surgery; CSR, clinical sales representative.

surgeons and one bedside surgeon.

Today, the training has evolved to include e-learning modules. Hands-on training courses are held at IRCAD in Strasbourg and EEC in Paris in France and at the ORSI Academy in Melle, Belgium. Two courses are identified: the beginner's course in which the surgical team is led through the ins and outs of the system and practices on porcine models to get "the feel of the console" while being guided by an experienced robotic console surgeon. Of course, the participants are allowed to work on the simulator as well as part of this course.

In the advanced course, trainees work on cadavers, with the objective of performing a thoracic procedure such as a lobectomy. Again, this course is led by a surgeon in order to focus on every day practice, troubleshooting and trouble solving.

This kind of training includes many positive and some negative aspects. It is time- consuming and intense training, often with travel from one country to the other but it allows the team members to be immersed in RATS, as Park et al. indicated. Before implementation of robotics into clinical practice, the surgeon and operating room team should attend an intensive, two-day certified course (10). Unfortunately, several items of the training are non- committal, which can lead to insufficient time spent doing dry-runs, practicing docking and emergency undocking and practicing at the simulator.

Case observations are facilitated by Intuitive but the frequency can be increased and it is more useful to organize a team-case observation rather than a surgeon-case observation.

Although there are now more proctors (eleven, including the United Kingdom) than in 2011, their availability and ability to commit to thorough training may vary due to their workload.

In all, training is a costly exercise and it is therefore important to get trained only in the context of a reputable program. The paragraph below summarizes several conditions that are necessary for implementation of RATS.

\section{A stepwise approach for a successful implementation of a RATS program}

\section{A. Requirements for institutions initiating a robotic program}

The Board of directors of the hospital as well as the staff of the thoracic surgery department should be supportive 
and willing to start the RATS program. Explicit collegial support of pulmonologists, cardiologists, anaesthetists and theater programmers is a prerequisite. There should be sufficient budget to acquire the latest generation surgical robot, preferably together with other disciplines such as urology, gynaecology, colorectal surgery or ear nose throat (ENT) in order to ascertain maximum use of the robot. A dual console unit, a simulator and a recording device should be an indispensable part of the business case to be used for proper teaching and training purposes. Basically, all the above-mentioned components can be summarized as "commitment".

This quote of the Ottawa Hospital is highly illustrative:

"The Ottawa Hospital/l'Hopital de Ottawa:

To expand the robotics program the Hospital must:

* Purchase upgraded technology for the da Vinci Surgical System.

* Provide the extensive technical support required to run an expanded program.

* Increase robotics simulation training to more surgeons at the Ottawa Skills and Simulation Centre at The Ottawa Hospital, so that more patients will benefit from this leading-edge surgical technology."

Lastly, we strongly recommend the institution should be part of a teaching hospital with residents who are in training for thoracic surgery.

\section{B. Requirements for the surgical teams}

The surgical team that is preparing to start a RATS program comprises two to three scrub and circulating nurses, one anaesthetist and two anaesthesia technicians, a dedicated bedside assistant and two surgeons that will be trained on the console. This dedicated team can be expanded later on in the process according to the number of patients that can be operated on annually.

It is definitely not recommended to have alternating team-members in the starting phase, as it will slow down the entire process with potential deleterious effects. The use of the simulator and review of recorded procedures should be encouraged.

Finally, the dedicated team and surgeons should have a broad exposure and experience in thoracic surgery and thoracic anesthesiology.

\section{Patient selection}

Patient selection is key when the program is just starting and although it is a costly issue to perform "simple cases" such as wedge resections, pleurectomy and bullectomy robotically, it shortens the learning curve and enhances technical skills and should therefore be allowed.

\section{Competencies for proctors}

At the moment, there are no special competencies required for proctorship, other than having RATS experience. Often the choice for the proctor who will assist the "starting" surgeon in the operating room is based on availability. Quite frequently the team and the proctor meet each other for the first time in the hospital where the program is about to start. Needless to say, this situation is open for improvement.

ISI distributes an evaluation form that is filled out by the trainees, but currently the proctor does not receive feedback on his teaching skills unless he actively asks for it.

One intangible factor is that the trainees and the proctor should speak the same language, literally as well as figuratively.

In June 2016 a working panel was created including members of the European Society of Thoracic Surgeons (ESTS) and European Association for Cardio-Thoracic Surgery (EACTS) with a specialist interest in robotic thoracic surgery with focus on training (11). An e-consensus-finding exercise using the Delphi methodology was applied, resulting in consensus that standardized robotic training should be divided in clearly defined sections as a staged learning pathway. The basic training is to include a baseline evaluation, an e-learning module and simulation training. The advanced training must include e-learning with video demonstration of index procedures such as right upper lobectomy, access to video library of robotic thoracic procedures, simulation training, modular console training, full mentored procedure training and a final evaluation of submitted video to be certified by independent examiners.

The proposal for a training program in Europe is largely based on the abovementioned study and touches on similar points as stated by Ricciardi et al. (12).

\section{Proposal for a training program}

(I) Ascertain a long-term plan to implement robotic thoracic surgery in daily practice;

(II) Provide a training program linked to a time path;

(III) Provide a mentor rather than a proctor;

(IV) Provide certification of knowledge and skills; 


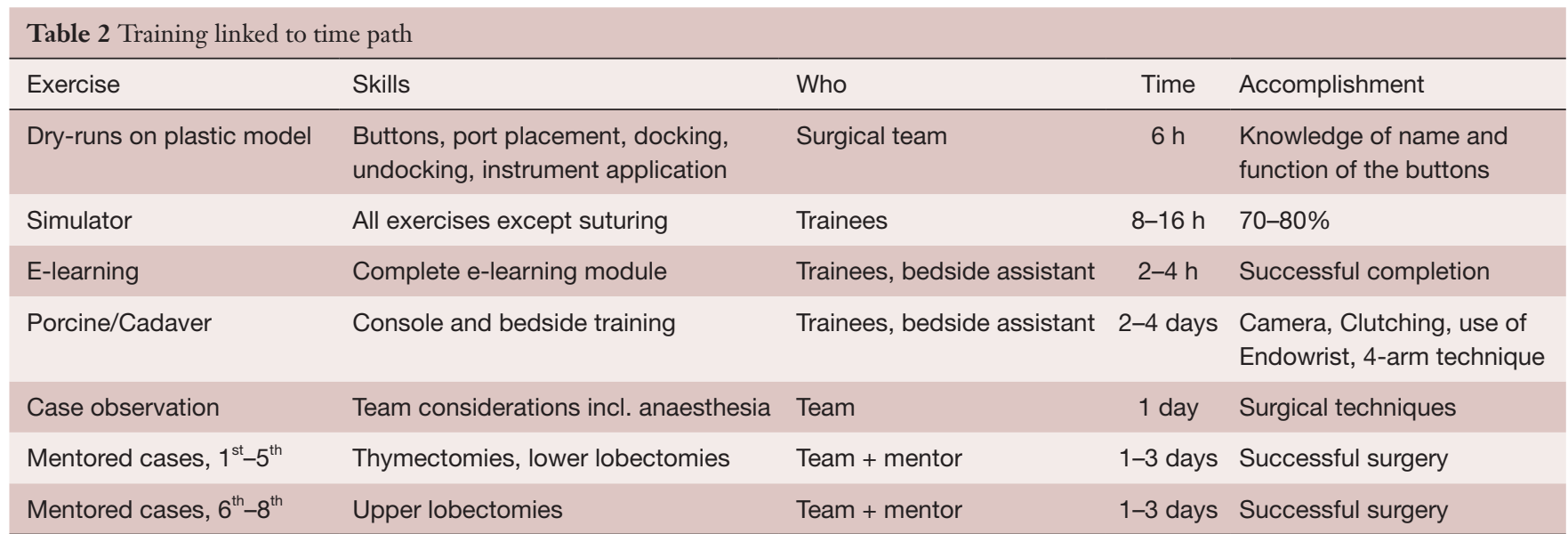

(V) Adequate evaluation of the experience after the teaching period is mandatory with bilateral feedback;

(VI) Data registration for research, quality surveillance and follow-up;

(VII) Next step for value-based health care centered on patients' well-being.

\section{(I) Ascertain a long-term plan to implement robotic thoracic surgery in daily practice}

If the goal is to implement robotic surgery as part of the surgical spectrum offered to patients, especially those with lung cancer, the best possible tailor-made treatment, a long-term plan or vision should be designed. From an economic point of view, it is important as well to ascertain that the robot will be used "24/7". Designated slots in the operation program can avoid frustrations concerning the availability of the robot and the dedicated surgery and anaesthesia team.

The fellows and residents of thoracic surgery, oncology and anesthesiology participate actively in the program to get acquainted with this technique right from the start. It is not necessary to have experience in VATS.

Ideally the surgeon, fellow or resident of thoracic surgery, who initially acts as a bedside assistant, progresses to being console surgeon. As several studies have shown the benefits of simulation $(13,14)$, we state that working on the simulator to achieve proficiency should be compulsory before the trainee is allowed to move to the console.

It is emphasized that thorough knowledge of the anatomy and surgical oncological principles is mandatory. The dual console system allows the trainee to act as console surgeon as long as an experienced console surgeon sits next to him for guidance throughout the operation. Initially, sections of the operation can be done and consequently proceed towards performing the entire operation. To achieve and maintain dexterity, the robotic skills must be practiced preferably every week. Having passed this initial learning curve, the aim should be at least 50 anatomic resections per year (15).

General infrastructure is mandatory and must include the instant availability of an intensive care unit (ICU).

\section{(II) Provide a training program linked to a time path}

We stumble on several difficulties when setting up a training curriculum linked to a time path, such as variability in surgical experience and dexterity, the availability of suitable patients, the availability of mentors and so on. However, by linking the program to a time path, a certain "flow" should be achieved to go from one competency level to another. It is our conviction that the learning curve depends on the amount of (successfully) performed procedures and on the timeframe the procedures are performed in. Undoubtedly, high volume specialized centres and high-volume surgeons have a positive impact on patient's outcome (16). Cerfolio has described in detail didactic routes and steps that should be followed to gain skills and proficiency in robotic major lung resections (17). The surgeon needs to become familiar with positioning of the robot, its arms and its instruments, the binocular and 3D-visualization, a restricted operative field, the handling of robotic surgical tools with a joystick (from a distance) and the absence of tactile feedback.

Table 2 summarizes the skills that must be exercised with 
an approximation of the time to be spent practicing. The exercises and the estimated duration necessary to become competent enough to move comfortably to the next level is merely based on our own experience and remains open for discussion.

Team training must include dry-runs with emphasis on patient positioning, port placement, docking and instrument placement. Knowledge of the buttonology must be practiced until "blindfolded" competency.

To achieve high quality robotic surgery, one should practice with the plastic model and follow an intensive 2-4-day surgeon led course. Ideally, the surgeon, fellow or resident of thoracic surgery, who initially acts as the bedside assistant, progresses to become console surgeon. Exercises for Camera \& Clutching, Endowrist ${ }^{\circledR}$ manipulation and Fourth Arm Integration on the skills simulator must be done to a level of at least $80 \%$.

A protocol for emergency conversion should be available and practiced.

Briefing and debriefing sessions must be held after each procedure, the first 5-7 in the presence of the proctor.

\section{(III) Provide a mentor rather than a proctor}

It is quite uncomfortable for a proctor to be confronted for the first time with a colleague he has never met before and of whom he is not informed about his level of skills and the level of the team. A proctor is partly responsible for the wellbeing of the patient. Therefore, we propose to apply the term mentor, as he should lead the porcine and cadaver courses, he provides the observation cases and who guides the beginning team through the initial learning curve. Thus, the mentor feels the commitment and responsibility to teach and train the team. The objective of the mentor is to facilitate the starting phase and lead it into a phase where robotic surgery is fully implemented in the surgical spectrum. It is therefore deemed fundamental that eligible mentoring surgeons in robotic thoracic surgery should be beyond the initial learning curve and perform at least 50 anatomic pulmonary resections per year. Side by side to having a reputation of high quality should be a compulsory training in teaching skills, such as a teach the teacher training. The mentor must also have insight in the feedback that the trainees give him in order to improve training skills.

Daily practice shows that it is hardly feasible to assign one mentor to one trainee. Given this, it might be wise to organize a proctor/mentor network where mentors can communicate but this is beyond the scope of this manuscript.

\section{(IV) Provide certification of knowledge and skills}

Items regarding the quality of a European training program need to be assessed and it is important that a scientific society (i.e., EACTS or ESTS) or a nested robotic thoracic working group should act as a certifying body for the training program. Submitted videos of several index procedures can be assessed by independent experts, which will lead to certification. Furthermore, assessment of competence at the end of the program by the associated mentor and quality monitoring after completion of the training program should be done.

It would sound logical that certification can be repeated periodically in order to maintain high quality.

\section{(V) Adequate evaluation of the experience after the teaching period is mandatory with bilateral feedback}

Debriefing sessions with the whole team and CSR are mandatory after every operation. A systematic evaluation of several operative items will be discussed (Table 3).

A similar feedback session should be held after the last mentored procedure but with the emphasis on the further steps to be taken to maintain a successful RATS program.

\section{(VI) Data registration for research, quality surveillance and follow-up}

Creating a database in which several patient characteristics and surgeon/surgery variables can be registered to monitor the patient outcome, surgical results and progression of skills and learning curve.

\section{(VII) Next step for value-based health care centred on patients well being}

With the minimally invasive surgical world being divided into pro-robot and pro-VATS surgeons and Intuitive Surgical Inc. being the company with the only available working robotic platform, the discussion will be centred around the costs of robotic surgery. Furthermore, there is no evidence of one technique surpassing the other as far as patient wellbeing is concerned. Creating a European database in which all robotic surgeons can register 


\begin{tabular}{|c|c|c|c|c|}
\hline Patient positioning & $x$ & & & $x$ \\
\hline Port placement & $x$ & & & $x$ \\
\hline Patient cart positioning & $x$ & & & $x$ \\
\hline Instrument application & & & $x$ & \\
\hline Use of camera & & $x$ & & \\
\hline Use of master manipulators & & $x$ & & \\
\hline Tissue handling & & $x$ & & \\
\hline Stapling & & $x$ & $\mathrm{x}$ & \\
\hline Suturing & & $x$ & & \\
\hline Specimen retrieval & & $x$ & $x$ & \\
\hline Steps and technique & & $x$ & $x$ & $x$ \\
\hline Ergonomics & $x$ & & & $x$ \\
\hline Troubleshooting \& solving & $x$ & & & $x$ \\
\hline Communication & $x$ & & & $x$ \\
\hline Feedback to proctor & $x$ & & & $x$ \\
\hline
\end{tabular}

their patients and results will allow profound research with enough patients to achieve statistical power. This is necessary to establish the value of robotic thoracic surgery for patients with malignancies of the lungs and mediastinum.

\section{Conclusions}

Robotic thoracic surgery can be taught to surgeons of varying levels of experience but this requires commitment of the trainee, the surgical team, anaesthesia, the mentor and the hospital administration.

In this article, we provided our experience and initiatives to establish a successful RATS program. Based on this experience and the most influential literature we proposed a structured training program for robotic thoracic surgery.

\section{Acknowledgements}

We thank Mrs. Roelien Kok for her contribution to this article.

\section{Footnote}

Conflicts of Interest: GM Shahin and S Stamenkovic are proctors for Intuitive Surgical Inc. The other authors have no conflicts of interest to declare.

\section{References}

1. Gossot D, Seguin-Givelet A. Video-assisted thoracic surgery (VATS) major pulmonary resections: different approaches and focus on the full thoraccoscopic fissurebased technique. Shangai Chest 2018;2:15

2. Reinersman JM, Passera E, Rocco G. Overview of uniportal video-assisted thoracic surgery (VATS): past and present. Ann Cardiothorac Surg 2016;5:112-7.

3. Carrott PW Jr, Jones DR. Teaching video-assisted thoracic surgery (VATS) lobectomy. J Thorac Dis 2013;5 Suppl 3:S207-11. 
4. Latif MJ, Park BJ. Robotics in general thoracic surgery procedures. J Vis Surg 2017;3:44.

5. Melfi FM, Mussi A. Robotically assisted lobectomy: learning curve and complications. Thorac Surg Clin 2008;18:289-95, vi-vii.

6. Kneuertz PJ, D'Souza DM, Moffatt-Bruce SD, et al. Robotic lobectomy has the greatest benefit in patients with marginal pulmonary function. J Cardiothorac Surg 2018;13:56.

7. Veronesi G, Park B, Cerfolio R, et al. Robotic resection of Stage III lung cancer: an international retrospective study. Eur J Cardiothorac Surg 2018;54:912-9.

8. Ruffini E, Filosso PL, Guerrera F, et al. Optimal surgical approach to thymic malignancies: New trends challenging old dogmas. Lung Cancer 2018;118:161-70.

9. Cerfolio RJ, Cichos KH, Wei B, et al. Robotic lobectomy can be taught while maintaining quality patient outcomes. J Thorac Cardiovasc Surg 2016;152:991-7.

10. Park BJ, Flores RM. Cost comparison of robotic, videoassisted thoracic surgery and thoracotomy approaches to pulmonary lobectomy. Thorac Surg Clin 2008; 18:297300, vii.

Cite this article as: Shahin GM, Brandon Bravo Bruinsma GJ, Stamenkovic S, Cuesta MA. Training in robotic thoracic surgery-the European way. Ann Cardiothorac Surg 2019;8(2):202-209. doi: 10.21037/acs.2018.11.06
11. Veronesi G, Dorn P, Dunning J, et al. Outcomes from the Delphi process of the Thoracic Robotic Curriculum Development Committee. Eur J Cardiothorac Surg 2018;53:1173-9.

12. Ricciardi S, Zirafa CC, Davini F, et al. How to get the best from robotic thoracic surgery. J Thorac Dis 2018;10:S947-50.

13. Culligan P, Gurshumov E, Lewis C, et al. Predictive validity of a training protocol using a robotic surgery simulator. Female Pelvic Med Reconstr Surg 2014;20:48-51.

14. Schreuder HW, Persson JE, Wolswijk RG, et al. Validation of a novel virtual reality simulator for robotic surgery. ScientificWorldJournal 2014;2014:507076.

15. Petersen RH, Hansen HJ. Learning curve associated with VATS lobectomy. Ann Cardiothorac Surg 2012;1:47-50.

16. Cheung MC, Hamilton K, Sherman R, et al. Impact of teaching facility status and high-volume centers on outcomes for lung cancer resection: an examination of 13,469 surgical patients. Ann Surg Oncol 2009;16:3-13.

17. Cerfolio RJ, Bryant AS. How to teach robotic pulmonary resection. Semin Thorac Cardiovasc Surg 2013;25:76-82. 